\title{
ADDI
}

Citation for published version (APA):

Allahgholi, M., Rahmani, H., Javdani, D., Weiss, G., \& Modos, D. (2020). ADDI: Recommending alternatives for drug-drug interactions with negative health effects. Computers in Biology and Medicine, 125, [103969]. https://doi.org/10.1016/j.compbiomed.2020.103969

Document status and date:

Published: 01/10/2020

DOI:

10.1016/j.compbiomed.2020.103969

Document Version:

Publisher's PDF, also known as Version of record

\section{Document license:}

Taverne

\section{Please check the document version of this publication:}

- A submitted manuscript is the version of the article upon submission and before peer-review. There can be important differences between the submitted version and the official published version of record.

People interested in the research are advised to contact the author for the final version of the publication, or visit the DOI to the publisher's website.

- The final author version and the galley proof are versions of the publication after peer review.

- The final published version features the final layout of the paper including the volume, issue and page numbers.

Link to publication

\footnotetext{
General rights rights.

- You may freely distribute the URL identifying the publication in the public portal. please follow below link for the End User Agreement:

www.umlib.nl/taverne-license

Take down policy

If you believe that this document breaches copyright please contact us at:

repository@maastrichtuniversity.nl

providing details and we will investigate your claim.
}

Copyright and moral rights for the publications made accessible in the public portal are retained by the authors and/or other copyright owners and it is a condition of accessing publications that users recognise and abide by the legal requirements associated with these

- Users may download and print one copy of any publication from the public portal for the purpose of private study or research.

- You may not further distribute the material or use it for any profit-making activity or commercial gain

If the publication is distributed under the terms of Article $25 \mathrm{fa}$ of the Dutch Copyright Act, indicated by the "Taverne" license above, 


\title{
ADDI: Recommending alternatives for drug-drug interactions with negative health effects
}

\author{
Milad Allahgholi ${ }^{a}$, Hossein Rahmani ${ }^{\text {a,* }}$, Delaram Javdani ${ }^{a}$, Gerhard Weiss ${ }^{\mathrm{b}}$, Dezső Módos ${ }^{\mathrm{c}, \mathrm{d}}$

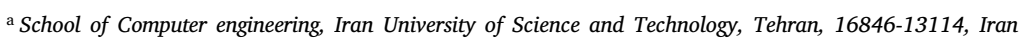 \\ ${ }^{\mathrm{b}}$ Maastricht University, PO Box 616, Maastricht 6200 MD, The Netherlands \\ ' Quadram Institute Bioscience, Norwich Research Park, Norwich, Norfolk, NR4 7UQ, UK \\ ${ }^{\mathrm{d}}$ Earlham Institute Norwich Research Park, Norwich, NR4 7UZ, UK
}

\section{A R T I C L E I N F O}

\section{Keywords:}

Alternative drug recommendation

Drug-drug interactions

Negative health effects

Deep learning

Word embedding

Text mining

\begin{abstract}
A B S T R A C T
Investigating the interactions among various drugs is an indispensable issue in the field of computational biology. Scientific literature represents a rich source for the retrieval of knowledge about the interactions between drugs. Predicting drug-drug interaction (DDI) types will help biologists to evade hazardous drug interactions and support them in discovering potential alternatives that increase therapeutic efficacy and reduce toxicity. In this paper, we propose a general-purpose method called ADDI (standing for Alternative Drug-Drug Interaction) that applies deep learning on PubMed abstracts to predict interaction types among drugs. As an application, ADDI recommends alternatives for drug-drug interactions (DDIs) which have Negative Health Effects Types (NHETs). ADDI clearly outperforms state-of-the-art methods, on average by $13 \%$, with respect to accuracy by using only the textual content of the online PubMed papers. Additionally, manual evaluation of ADDI indicates high precision in recommending alternatives for DDIs with NHETs.
\end{abstract}

\section{Introduction}

Lately, discovering interactions among drugs attracted a lot of attention [1-8]. Biological literature is a rich source of knowledge for discovering interactions among drugs [9-14] and this gives computational text mining approaches an important role in current drug studies [1517]. The intended effect of a drug can be changed with the simultaneous use of another drug [18]. There are varied definitions for druginteraction with respect to different perspectives/vocabularies/etc. Some definitions for drug interactions are:

- "A drug interaction is a change in the action or side effects of a drug caused by concomitant administration with a food, beverage, supplement, or another drug" [19].

- "A drug interaction has occurred when the administration of one drug alters the clinical effects of another. The result may be an increase or decrease in either the beneficial or harmful effects of the second agent" [20].

- "A drug interaction can be defined as an interaction between a drug and another substance that prevents the drug from performing as expected" [21].

- "One drug can affect the activity of another when they are administered together, which can cause adverse drug reactions or sometimes improve therapeutic effects" [22].
- "A drug interaction occurs when a substance affects the activity of a drug, either increasing or decreasing its efficacy, or, alternatively, a new effect is observed that is not observed with just the drug alone" [23].

Understanding types of DDIs is essential to recommend alternatives that decrease unexpected adverse drug events (ADEs) and increase synergistic advantages [24-26].

Available computational methods for predicting DDIs are usually based on structural and other similarities or drug-target associations [27-34]. In the following, we refer to selected representatives of these related approaches. Cheng et al. [35] generated a drug-drug network by quantifying the relationships between drug targets and disease proteins in the human protein-protein interactome to predict clinically useful drug combinations for specific diseases. Rohani and Eslahchi [32] proposed a neural network-based method for DDI prediction that applied the neural network model with similarity selection and fusion methods to increase the accuracy of predicting DDIs. Qian et al. [36] constructed a gradient boosting-based classifier to predict adverse DDIs using genetic interactions between the gene targets of two drugs. They discovered that adversely interacting drug targets are more likely to have more synergistic genetic interactions than noninteracting drugs targets. Karim et al. [37] combined a convolutional

\footnotetext{
* Corresponding author.

E-mail address: h_rahmani@iust.ac.ir (H. Rahmani).
} 


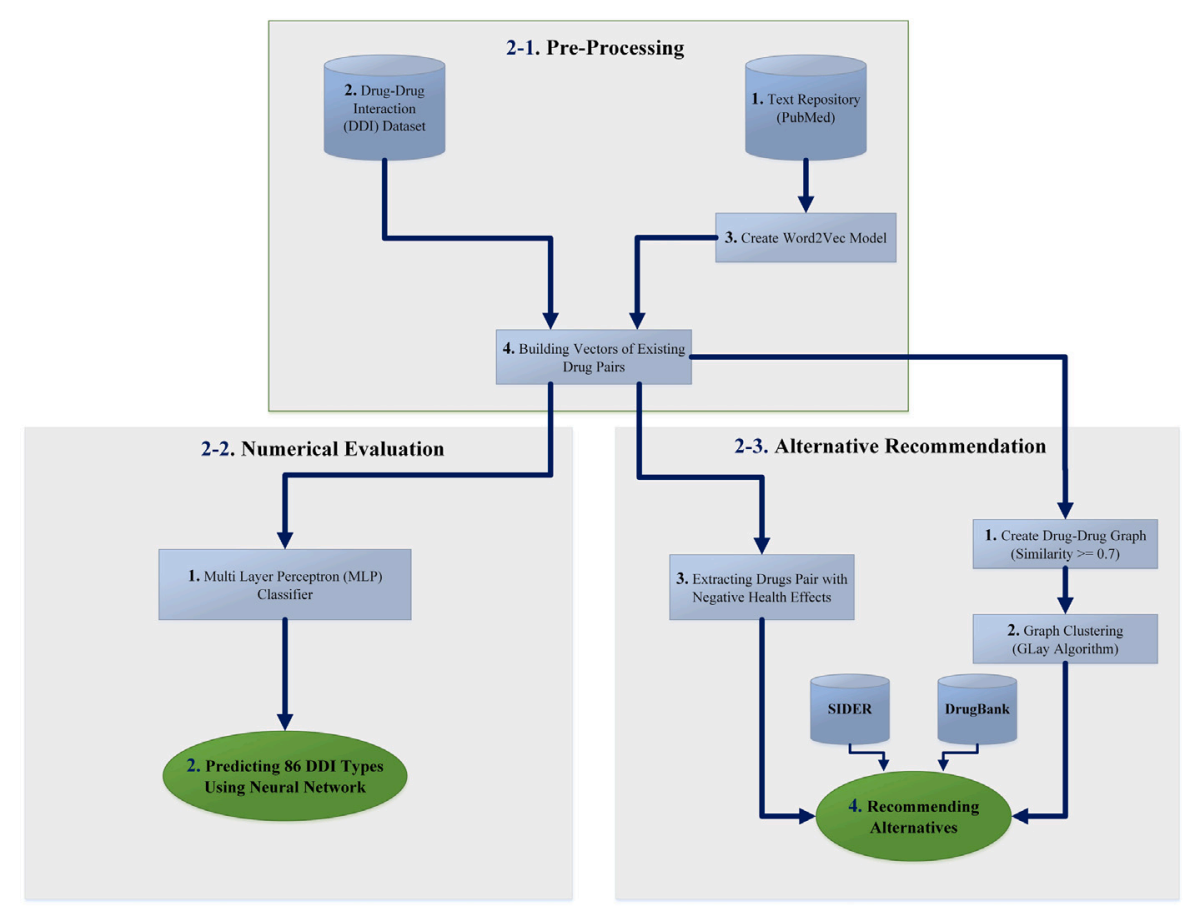

Fig. 1. Overview of ADDI method.

neural network (CNN) and a long short-term memory (LSTM) network to predict DDIs in multiple data sources that are integrated using Knowledge Graphs. They embed the nodes in the graph applying various embedding approaches. Ryu et al. [24] proposed a computational framework, DeepDDI, that takes chemical structural information of two drugs as inputs and accurately predicts relevant DDI types for the input drug pair.

The main drawback of previous methods for predicting DDIs is that they require detailed drug information such as the chemical structure of drugs, drug targets or side effects as input, which are often error-prone and costly and time-consuming to provide. As an alternative to these methods, there are methods that exploit text-mining to extract DDIs using less information. Huang et al. used LSTM and POS embedding to extract features from the text in order to predict DDIs [38]. Zhao et al. proposed a syntax convolutional neural network (SCNN) based DDI extraction method [39]. Lim et al. used PubMed-and-PMC-w2v and LSTM to extract DDIs [40]. Shi et al. used matrix factorization to predict drugdrug interactions (DDIs) [41]. They used drug-binding proteins. Zheng et al. proposed a method called DDI-PULearn that predicted DDIs using SVM and KNN algorithms as well as using the PCA feature reduction method [42]. All of these text-mining approaches apply pre-trained models on only one kind of text input and are limited in their extension capacity. In this paper, we propose an expandable method called ADDI (Alternative Drug-Drug Interactions) that uses online text resources such as PubMed to predict interaction types among drugs. ADDI applies a word-embedding technique known as Word2vec [43,44] on 29 million PubMed paper abstracts [45] to extract drug embeddings. ADDI uses a neural network that receives drug embeddings as input to predict DDI types as output. Additionally, ADDI creates a drug-drug network. ADDI applies a clustering algorithm to recommend alternatives for DDIs which have negative health effects types (NHETs). The key contribution of this paper is:

- ADDI is the first and still informative method in combining most recent advanced deep learning-based text mining approaches with graph mining approaches for the task of recommending novel alternatives for drug pairs with negative health effects. Additionally, compared to the state-of-the-art methods, ADDI
Table 1

Parameters configuration of Word2Vec.

\begin{tabular}{ll}
\hline Parameter & Setting \\
\hline Model & CBOW \\
min-count & 5 \\
dim & 200 \\
samp & $1 \mathrm{e}-4$ \\
win & 8 \\
\hline
\end{tabular}

produces more accurate results by using less amount of information (only online PubMed texts) as input data. The manual evaluation of recommended alternatives indicates the predictive power of ADDI.

The structure of this paper is as follows: ADDI is described in detail in Section 2. The empirical results are presented in Section 3 and discussed in Section 4. Section 5 summarizes major insights and proposes promising directions for future research.

\section{ADDI}

The basic idea underlying ADDI is to apply deep learning to discover DDI types and to recommend alternatives for interactions with negative health effects. The main steps of ADDI are shown in Fig. 1, and the following subsections will discuss each main step in detail.

\subsection{Pre-processing}

Pre-processing consists of the following four sub-steps through which the input data are polished and integrated so that they can be further processed in the subsequent main steps "Numerical Evaluation" and "Application":

1. ADDI is a general method that works on textual data that contains relevant information about drugs and DDIs. For this purpose, ADDI employed two datasets in the experimental analysis described in Section 3. As a text repository containing drug information, ADDI uses the PubMed dataset which contains all 
Table 2

14 DDI types known as Negative Health Effects.

\begin{tabular}{ll}
\hline Index & Negative Health Effects \\
\hline 1 & Cardiotoxicity \\
2 & Central neurotoxicity \\
3 & Hepatotoxicity \\
4 & Nephrotoxicity \\
5 & Neurotoxicity \\
6 & Ototoxicity \\
7 & Hypersensitivity \\
8 & Adverse effects \\
9 & Bleeding \\
10 & Heart failure \\
11 & Hyperkalemia \\
12 & Hypertension \\
13 & Hypotension \\
14 & QTc prolongation \\
\hline
\end{tabular}

the information regarding PubMed papers, such as title, abstract, authors' name, year of publication, etc. ADDI extract abstracts of all papers in the PubMed dataset. This resulted in a repository of 30 GB data, which we made available in [46]. ADDI constructs word2vec embedding for each drug term using the PubMed dataset. These embeddings are considered as input features of multilayer perceptron (MLP) network [47] (discussed later in Section 2.2).

2. ADDI requires data that comprises DDIs. For the experiments reported in Section 3, ADDI used the drug-drug interaction (DDI) dataset described in [24], which contains 192,284 DDIs representing 86 DDI types. Each DDI represents a zero-one feature $D D I_{i, j}^{t_{k}}$ indicating whether DDI type $t_{k}$ existed between two drugs $d_{i}$ and $d_{j}$. These features are used in the output layer of the MLP network (discussed later in Section 2.2). The list of all 86 DDI types is available at $[48,49]$. DDI types describe changes in pharmacological effects and the risk of adverse drug events as a result of the interaction between two drugs in pairs [24]. For instance, one drug may alter the pharmacokinetics of another. Among the 86 DDI types, 14 DDI types are known to be classed as "Negative Health Effects" (see Table 2). The drug pairs having "negative health effects" are those which are described with "the increased risk or severity of adverse effects" and explicit expression such as "cardiotoxic activity", "nephrotoxic activity", and "the increased risk or severity of bleeding".

3. ADDI applies Word2Vec $[44,50]$ on the PubMed dataset to describe each drug term in PubMed. Word2Vec is a two-layer neural network that processes text by describing each word in the text with a small vector. Word2vec takes as its input a text corpus and produces a set of feature vectors that represent words in that corpus [50]. CBOW and Skip-gram are two Word2vec models. Skip-gram and CBOW are more efficient in the case of smaller and larger volumes of training data, respectively [43]. When running ADDI with $30 \mathrm{~GB}$ of data (which is considered as a high volume of data), we expected that $\mathrm{CBOW}$ would discover more DDIs than skip-gram. The configuration of Word2Vec is shown in Table 1 . The minimum count (min-count) specifies the minimum number of occurrences needed for a word to be included in the word vectors. The vector dimension (dim) is the learned word vector size. High-frequency words usually provide little information. Sub-sampling (samp) is the process of diminishing frequent word occurrences (words with a frequency above a certain threshold) in order to increase training speed. The context window size (win) denotes the range of words to be included as the target word context [51-53]. The values used in our experiments for these parameters are shown in Table 1 and have been chosen according to best practices described in the literature [44,51,54-58].

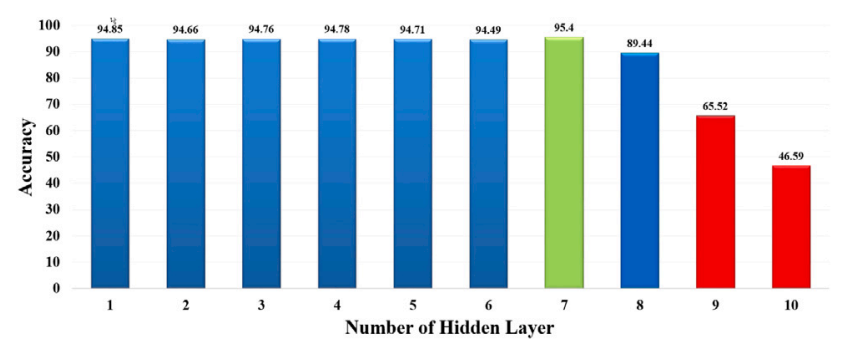

Fig. 2. Accuracy of MLP network in varied hidden layers. Accuracy maximizes when the number of hidden layers equals 7. MLP network overfits when the number of hidden layers exceeds 8 .

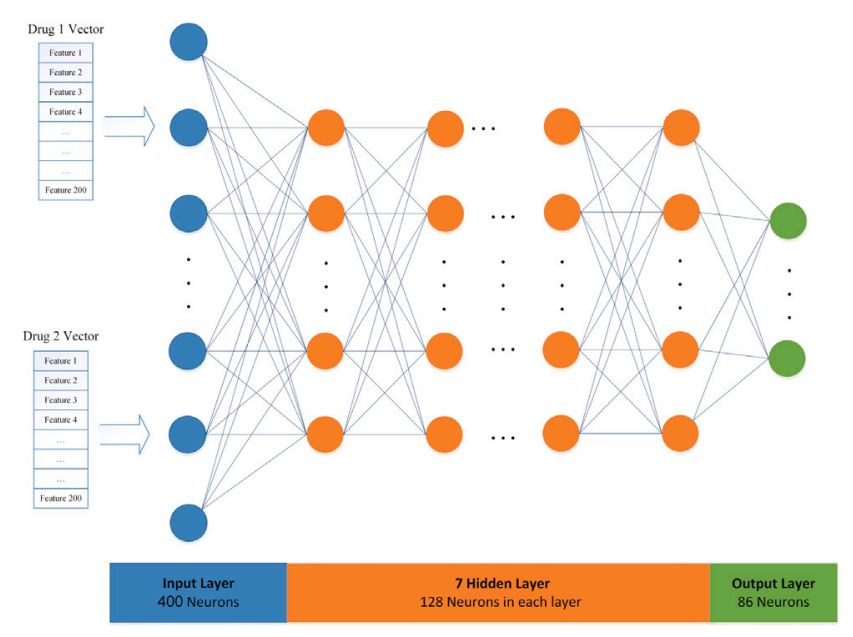

Fig. 3. An illustration of the MLP neural network used by the ADDI method.

4. ADDI predicts DDIs using drug embeddings extracted by Word2Vec from our text repository (For each drug term $\left(d_{i}\right)$ in PubMed dataset, Word2vec extracts a vector $\left.\left(\overline{W 2 V}\left(d_{i}\right)\right)\right)$. Therefore, from all the DDIs in the DDI dataset, we only considered the DDIs in which the involved drugs exist in our Word2Vec model.

\subsection{Numerical evaluation}

ADDI utilizes neural networks $[59,60]$ to predict the type of interaction among drug pairs. These networks consist of multiple layers of simple processing units called neurons, where each such unit generates an output signal based its input signals according to some activation function. Neural networks have been shown in various domains to be extremely powerful pattern recognition tools $[59,60]$.

1. Specifically, for the experiments reported here, Multilayer Perceptrons (MLP) was used [47]. This is a class of feedforward artificial neural network that has the following architecture:

- The input layer contains 400 neurons (200 dimensions $\overrightarrow{W 2 V}\left(d_{i}\right)+200$ dimensions $\left.\overrightarrow{W 2 V\left(d_{j}\right)}\right)$.

- The output layer contains 86 neurons, one for each of the 86 DDI types in the DDI dataset (see Section 2.1).

- Neurons' activation functions, Sigmoid [61] and Softmax [62], are used in the hidden layers and in the output layer, respectively.

- To determine the number of hidden layers, we examined the MLP network with a varied range of hidden layers, calculating accuracy using a 10-fold cross validation. As shown in Fig. 2, the MLP network reaches its best accuracy when the number of hidden layers equals 7. It was also observed that with more 
Table 3

The result of ADDI compared to state-of-the-art methods.

\begin{tabular}{lll}
\hline Methods & Type & Accuracy \\
\hline ADDI & Text-mining & $\mathbf{9 5 . 4 \%}$ \\
Ryu et al. [24] & Chemical structures & $92.4 \%$ \\
Huang et al. [38] & Text-mining & $69 \%$ \\
Zhao et al. [39] & Text-mining & $68.9 \%$ \\
Lim et al. [40] & Text-mining & $83.8 \%$ \\
Shi et al. [41] & Text-mining & $90 \%$ \\
Zheng et al. [42] & Text-mining & $90.4 \%$ \\
\hline
\end{tabular}

than 8 layers, the MLP network overfits to the training data and produced a worse result.

An illustration of the MLP neural network is shown in Fig. 3. The network consists of an input layer with 400 neurons, seven hidden layers each having 128 neurons, and output layer with 86 neurons.

2. ADDI utilizes the MLP neural network to predict DDIs and employs 10 -fold cross-validation for evaluation and comparing itself with previous methods.

\subsection{Alternative recommendation}

ADDI recommends alternatives for drug pairs with negative health effects by executing the following four steps:

1. For all $\left(d_{i}, d_{j}\right)$, ADDI uses the cosine similarity between $\overrightarrow{W 2 V}\left(d_{i}\right)$ and $\overrightarrow{W 2 V}\left(d_{j}\right)$ to calculate their semantic similarity (see Formula (1)):

$\operatorname{SemSim}\left(d_{i}, d_{j}\right)=\frac{\overrightarrow{W 2 V}\left(d_{i}\right) \cdot \overrightarrow{W 2 V}\left(d_{j}\right)}{\left\|\overrightarrow{W 2 V}\left(d_{i}\right)\right\| \times\left\|\overrightarrow{W 2 V}\left(d_{j}\right)\right\|}$

where $\overrightarrow{W 2 V}\left(d_{i}\right) \cdot \overrightarrow{W 2 V}\left(d_{j}\right)$ is the dot product of the Word2Vec vectors of $d_{i}$ and $d_{j}$ and $\left\|\overrightarrow{W 2 V}\left(d_{i}\right)\right\|$ stands for the magnitude of that vector. $\operatorname{Sem} \operatorname{Sim}\left(d_{i}, d_{j}\right)$ indicates the semantic similarity of two drugs $d_{i}$ and $d_{j}$ with respect to their Word2Vec models. ADDI considers only the most informative discovered interactions between drug pairs by pruning the interactions with semantic similarity values less than $\gamma_{1}$. More precisely, an interaction between two drugs $d_{i}$ and $d_{j}\left(D D I\left(d_{i}, d_{j}\right)\right)$ is pruned if $\operatorname{SemSim}\left(d_{i}, d_{j}\right)<\gamma_{1}$ (see Formula (2)):

$\operatorname{Pruning}\left(D D I\left(d_{i}, d_{j}\right)\right)= \begin{cases}\operatorname{remove}\left(D D I\left(d_{i}, d_{j}\right)\right), & \text { if } \operatorname{SemSim}\left(d_{i}, d_{j}\right)<\gamma_{1} \\ \operatorname{keep}\left(D D I\left(d_{i}, d_{j}\right)\right), & \text { otherwise }\end{cases}$

For the experiments reported here, $\gamma_{1}=0.7$ was chosen. In our studies, we found that $\gamma_{1}=0.7$ is high enough to exclude most irrelevant interactions and low enough to prevent loss of possibly relevant interactions.

ADDI constructs a graph $G(V, E)$ of the DDIs in which each node $v_{i} \in V$ represents a drug $d_{i}$ and each edge $e_{i j} \in E$ indicates that the semantic similarity (see Formula (1)) between the interacting drugs $d_{i}$ and $d_{j}$ is greater than 0.7.

2. ADDI applies the Glay algorithm [63] to cluster the graph of DDIs. This algorithm partitions the graph $G$ into $\mathrm{m}$ nonoverlapping clusters $c_{1}, \ldots, c_{m}$. If $d_{i}$ is the member of cluster $c_{k}$ $(1 \leq k \leq m)$ then CoMemCluster $\left(d_{i}\right)$ returns the set of drugs belonging to same cluster as drug $d_{i}$. Formally this is expressed by Formula (3)

CoMemCluster $\left(d_{i}\right)=\bigcup_{d_{j} \in D}\left(\operatorname{cluster}\left(d_{i}\right)=\operatorname{cluster}\left(d_{j}\right)\right)$

where $D$ is the set of all drugs and cluster $\left(d_{i}\right)$ returns the index of cluster of $d_{i}$. CoMemCluster $\left(d_{i}\right)$ indicates a set of drugs that are in the same cluster of drug $d_{i}$ and accordingly, are contextually similar to each other with respect to their appearance in the biological text repository.

3. Among the 86 DDI types in DDI dataset, 14 DDI types are known to be of the "Negative Health Effects" type (see Table 2). ADDI extracts 180 drug pairs with these 14 DDI types in order to recommend alternatives. In the rest of this paper, we refer to these 14 DDI types with Negative Health Effects as "NHETs".

4. ADDI uses the result of clustering to reduce the search space for recommending alternative drugs. For this purpose, if an interaction between drugs $d_{i}$ and $d_{j}$ is among the NHETs then ADDI aims to discover all alternative drugs $d_{i}$, for drug $d_{i}$ that fulfill the following three conditions:

(1) $d_{i}, \in$ CoMemCluster $\left(d_{i}\right)$

(2) $d_{i \prime}$, individually, does not have negative health effects (ADDI uses the SIDER dataset ${ }^{1}$ to consider each drug's side-effect individually and exclude drugs that cause negative health effects. SIDER contains information on marketed medicines and their recorded adverse drug reactions.)

(3) DDITypes $\left(d_{i}, d_{j}\right) \notin$ NHETs (To eliminate drug pairs that have NHETs, ADDI utilizes DrugBank dataset. ${ }^{2}$ The DrugBank database is a unique bioinformatics and cheminformatics resource that combines detailed drug data with comprehensive drug target information.)

where DDITypes $\left(d_{i}, d_{j}\right)$ is interaction type between two drugs $d_{i}$ and $d_{j}$. In other words, ADDI examines the drugs that are in the same cluster as $d_{i}$, looking for all drugs $d_{i^{\prime}}$ that do not have negative health effects individually and in combination with drug $d_{j}$. In exactly the same way ADDI aims to discover all alternative drugs $d_{j^{\prime}}$ for $d_{j}$ that have no negative effects with drug $d_{i}$.

For all discovered alternative drugs $d_{i}$, and $d_{j}$, ADDI calculates the semantic similarities $\operatorname{SemSim}\left(d_{i}, d_{i^{\prime}}\right)$ and $\operatorname{SemSim}$ $\left(d_{j}, d_{j^{\prime}}\right)$, respectively. In a last step, ADDI is looking for (i) $d_{i^{\prime}}^{\text {MaxSim }}$ as an alternative for $d_{i}$ so that $\operatorname{Sem} \operatorname{Sim}\left(d_{i}, X\right)$ is highest for $\mathrm{X}=d_{i^{\prime}}^{\text {MaxSim }}$ and (ii) $d_{j^{\prime}}^{\text {MaxSim }}$ as an alternative for $d_{j}$ so that $\operatorname{Sem} \operatorname{Sim}\left(d_{j}, Y\right)$ is highest for $\mathrm{Y}=d_{j^{\prime}}^{\operatorname{Max} \operatorname{Sim}}$. Finally, ADDI recommends three alternatives for $D D I\left(d_{i}, d_{j}\right)$ :

$$
\begin{aligned}
& \text { (1) } D D I\left(d_{i^{\prime}}^{\text {MaxSim }}, d_{j}\right) \\
& \text { (2) } D D I\left(d_{i}, d_{j^{\prime}}^{\text {MaxSim }}\right) \\
& \text { (3) DDI }\left(d_{i^{\prime}}^{\text {MaxSim }}, d_{j^{\prime}}^{\text {MaxSim }}\right) \text { where } \\
& \text { DDITypes }\left(d_{i^{\prime}}^{\text {MaxSim }}, d_{j^{\prime}}^{\text {MaxSim }}\right) \notin \text { NHETs. }
\end{aligned}
$$

Among all the drugs $d_{i^{\prime}}$ that 1- Are in the same cluster of drug $d_{i}$ and 2- Have no negative health effect in interacting with drug $d_{j}$, $\operatorname{DDI}\left(d_{i^{\prime}}^{\text {MaxSim }}, d_{j}\right)$ returns $d_{i^{\prime}}^{\text {MaxSim }}$ that have the maximum contextual similarity with drug $d_{i}$. In other words, $D D I\left(d_{i^{\prime}}^{\text {MaxSim }}, d_{j}\right)$ returns an alternative drug that is biologically very similar to drug $d_{i}$ and can replace drug $d_{i}$ and more importantly, does not have negative health effect interaction with drug $d_{j}$. In some cases, It is better to replace both drug $d_{i}$ and drug $d_{j}$ in the drug combination. Among all the drugs $d_{i^{\prime}}$ in the same cluster as $d_{i}$ and among all the drugs $d_{j^{\prime}}$ in the same cluster of drug $d_{j}, D D I\left(d_{i^{\prime}}^{\text {MaxSim }}, d_{j^{\prime}}^{\text {MaxSim }}\right)$ recommends drug pair $\left(d_{i^{\prime}}, d_{j^{\prime}}\right)$ with the maximum contextual similarity as an alternative recommendation for drug pair $\left(d_{i}, d_{j}\right)$.

\section{Empirical results}

In the following subsections, we first evaluate the predicted DDI types and then demonstrate the ability of ADDI to recommend alternatives for DDIs with NHETs.

\footnotetext{
1 http://sideeffects.embl.de/.

2 https://www.drugbank.ca/.
} 
Table 4

99 DDIs with NHE-type and their alternatives.

\begin{tabular}{|c|c|c|c|c|c|c|c|}
\hline Index & $d_{1}$ & $d_{2}$ & $d_{1^{\prime}}^{\operatorname{MaxSim}}$ & $d_{2^{\prime}}^{M a x \operatorname{Sim}}$ & $\operatorname{SemSim}\left(d_{1}, d_{1^{\prime}}^{\text {MaxSim }}\right)$ & $\operatorname{SemSim}\left(d_{2}, d_{2^{\prime}}^{\operatorname{MaxSim}}\right)$ & NHETs \\
\hline 1 & dofetilide & escitalopram & flecainide & agomelatine & 0.769 & 0.704 & QTc prolongation \\
\hline 2 & ibutilide & escitalopram & flecainide & agomelatine & 0.76 & 0.704 & QTc prolongation \\
\hline 3 & sotalol & escitalopram & flecainide & agomelatine & 0.803 & 0.704 & QTc prolongation \\
\hline 4 & ibutilide & lopinavir & dofetilide & amprenavir & 0.759 & 0.733 & QTc prolongation \\
\hline 5 & dofetilide & disopyramide & flecainide & aprindine & 0.769 & 0.794 & QTc prolongation \\
\hline 6 & dofetilide & thioridazine & flecainide & chlorpromazine & 0.769 & 0.711 & QTc prolongation \\
\hline 7 & ibutilide & amiodarone & flecainide & flecainide & 0.76 & 0.755 & QTc prolongation \\
\hline 8 & dofetilide & ibutilide & flecainide & flecainide & 0.769 & 0.76 & QTc prolongation \\
\hline 9 & dofetilide & sotalol & flecainide & flecainide & 0.769 & 0.803 & QTc prolongation \\
\hline 10 & ibutilide & sotalol & flecainide & flecainide & 0.76 & 0.803 & QTc prolongation \\
\hline 11 & dofetilide & pimozide & flecainide & fluphenazine & 0.769 & 0.713 & QTc prolongation \\
\hline 12 & ibutilide & pimozide & flecainide & fluphenazine & 0.76 & 0.713 & QTc prolongation \\
\hline 13 & sotalol & pimozide & flecainide & fluphenazine & 0.803 & 0.713 & QTc prolongation \\
\hline 14 & dofetilide & zuclopenthixol & flecainide & fluphenazine & 0.769 & 0.73 & QTC prolongation \\
\hline 15 & ibutilide & zuclopenthixol & flecainide & fluphenazine & 0.76 & 0.73 & QTc prolongation \\
\hline 16 & sotalol & zuclopenthixol & flecainide & fluphenazine & 0.803 & 0.73 & QTc prolongation \\
\hline 17 & dofetilide & quinidine & flecainide & mexiletine & 0.769 & 0.732 & QTc prolongation \\
\hline 18 & ibutilide & quinidine & flecainide & mexiletine & 0.76 & 0.732 & QTc prolongation \\
\hline 19 & sotalol & quinidine & flecainide & mexiletine & 0.803 & 0.732 & QTc prolongation \\
\hline 20 & dofetilide & flupentixol & mexiletine & perphenazine & 0.704 & 0.779 & QTc prolongation \\
\hline 21 & sotalol & flupentixol & mexiletine & perphenazine & 0.734 & 0.779 & QTc prolongation \\
\hline 22 & ibutilide & nilotinib & flecainide & ponatinib & 0.76 & 0.771 & QTc prolongation \\
\hline 23 & sotalol & nilotinib & flecainide & ponatinib & 0.803 & 0.771 & QTc prolongation \\
\hline 24 & dofetilide & citalopram & flecainide & reboxetine & 0.769 & 0.731 & QTc prolongation \\
\hline 25 & sotalol & lopinavir & dofetilide & ritonavir & 0.796 & 0.748 & QTc prolongation \\
\hline 26 & dofetilide & iloperidone & flecainide & sertindole & 0.769 & 0.753 & QTc prolongation \\
\hline 27 & ibutilide & iloperidone & flecainide & sertindole & 0.76 & 0.753 & QTc prolongation \\
\hline 28 & sotalol & iloperidone & flecainide & sertindole & 0.803 & 0.753 & QTc prolongation \\
\hline 29 & dofetilide & cisapride & flecainide & tegaserod & 0.769 & 0.725 & QTc prolongation \\
\hline 30 & ibutilide & cisapride & flecainide & tegaserod & 0.76 & 0.725 & QTc prolongation \\
\hline 31 & sotalol & cisapride & flecainide & tegaserod & 0.803 & 0.725 & QTc prolongation \\
\hline 32 & ibutilide & asenapine & dronedaron€ & vilazodone & 0.704 & 0.718 & QTc prolongation \\
\hline 33 & dofetilide & asenapine & mexiletine & vilazodone & 0.704 & 0.718 & QTc prolongation \\
\hline 34 & sotalol & asenapine & mexiletine & vilazodone & 0.734 & 0.718 & QTc prolongation \\
\hline 35 & vemurafenib & minaprine & erlotinib & amineptine & 0.729 & 0.727 & QTc prolongation \\
\hline 36 & vemurafenib & dapiprazole & erlotinib & apraclonidine & 0.729 & 0.772 & QTc prolongation \\
\hline 37 & vemurafenib & gatifloxacin & erlotinib & besifloxacin & 0.729 & 0.723 & QTc prolongation \\
\hline 38 & vemurafenib & pirbuterol & erlotinib & bitolterol & 0.729 & 0.757 & QTc prolongation \\
\hline 39 & vemurafenib & domperidone & erlotinib & cisapride & 0.729 & 0.726 & QTc prolongation \\
\hline 40 & vemurafenib & paroxetine & erlotinib & clomipramine & 0.729 & 0.78 & QTc prolongation \\
\hline 41 & vemurafenib & flecainide & erlotinib & dofetilide & 0.729 & 0.769 & QTc prolongation \\
\hline 42 & vemurafenib & sotalol & erlotinib & dofetilide & 0.729 & 0.796 & QTc prolongation \\
\hline 43 & vemurafenib & milnacipran & erlotinib & duloxetine & 0.729 & 0.769 & QTc prolongation \\
\hline 44 & vemurafenib & amoxapine & erlotinib & maprotiline & 0.729 & 0.796 & QTc prolongation \\
\hline 45 & vemurafenib & isocarboxazid & erlotinib & maprotiline & 0.729 & 0.707 & QTc prolongation \\
\hline 46 & vemurafenib & phenelzine & erlotinib & maprotiline & 0.729 & 0.714 & QTc prolongation \\
\hline 47 & vemurafenib & protriptyline & erlotinib & maprotiline & 0.729 & 0.772 & QTc prolongation \\
\hline 48 & vemurafenib & prochlorperazine & erlotinib & metoclopramide & 0.729 & 0.735 & QTc prolongation \\
\hline 49 & vemurafenib & fluconazole & erlotinib & micafungin & 0.729 & 0.718 & QTc prolongation \\
\hline 50 & vemurafenib & clotrimazole & erlotinib & miconazole & 0.729 & 0.752 & QTc prolongation \\
\hline 51 & vemurafenib & econazole & erlotinib & miconazole & 0.729 & 0.811 & QTc prolongation \\
\hline 52 & vemurafenib & sertaconazole & erlotinib & miconazole & 0.729 & 0.76 & QTc prolongation \\
\hline 53 & vemurafenib & moclobemide & erlotinib & nefazodone & 0.729 & 0.787 & QTc prolongation \\
\hline 54 & vemurafenib & tranylcypromine & erlotinib & pargyline & 0.729 & 0.731 & QTc prolongation \\
\hline 55 & vemurafenib & fluphenazine & erlotinib & perphenazine & 0.729 & 0.806 & QTc prolongation \\
\hline 56 & vemurafenib & molindone & erlotinib & perphenazine & 0.729 & 0.763 & QTc prolongation \\
\hline 57 & vemurafenib & zuclopenthixol & erlotinib & perphenazine & 0.729 & 0.725 & QTC prolongation \\
\hline 58 & vemurafenib & nalbuphine & erlotinib & pethidine & 0.729 & 0.732 & QTc prolongation \\
\hline 59 & vemurafenib & mesoridazine & erlotinib & promazine & 0.729 & 0.75 & QTc prolongation \\
\hline
\end{tabular}

(continued on next page)

\subsection{Predicting DDI types}

ADDI accepts the Word2Vec of two drugs as an input of a neural network (as described in Section 2.2) and predicts the interaction types of two input drugs as an output of the neural network. We made the structure of the MLP algorithm available at [49]. Comparing ADDI to state-of-the-art methods, ADDI outperforms those methods with respect to accuracy (see Table 3). Accuracy is defined as (see Formula (4)):

Accuracy $=\frac{\text { Number of Correct (Predicted) DDIs }}{\text { Number of existed DDIs (in Dataset) }}$
This is remarkable because ADDI succeeded in outperforming both text mining-based [38-40] and non-text mining-based [24] approaches. In particular, compared to Ryu et al. [24], which receives chemical structures of two drugs as input, ADDI generates an equally accurate (and even slightly better) result with significantly less information it only uses the information available online in the PubMed dataset.

\subsection{Recommending alternatives}

A key feature of ADDI is its ability to recommend alternatives for DDIs with NHETs. As described in Section 2, this ability is based on 
Table 4 (continued).

\begin{tabular}{|c|c|c|c|c|c|c|c|}
\hline Index & $d_{1}$ & $d_{2}$ & $d_{1^{\prime}}$ & $d_{2^{\prime}}$ & $\operatorname{SemSim}\left(d_{1}, d_{1^{\prime}}\right)$ & $\operatorname{SemSim}\left(d_{2}, d_{2^{\prime}}\right)$ & NHETs \\
\hline 60 & vemurafenib & triflupromazine & erlotinib & promazine & 0.729 & 0.834 & QTC prolongation \\
\hline 61 & vemurafenib & remoxipride & erlotinib & sertindole & 0.729 & 0.743 & QTC prolongation \\
\hline 62 & vemurafenib & dezocine & erlotinib & sufentanil & 0.729 & 0.721 & QTc prolongation \\
\hline 63 & vemurafenib & remifentanil & erlotinib & sufentanil & 0.729 & 0.784 & QTc prolongation \\
\hline 64 & vemurafenib & progabide & erlotinib & tiagabine & 0.729 & 0.753 & QTc prolongation \\
\hline 65 & vemurafenib & terconazole & erlotinib & tioconazole & 0.729 & 0.735 & QTc prolongation \\
\hline 66 & vemurafenib & bifonazole & erlotinib & tolnaftate & 0.729 & 0.825 & QTc prolongation \\
\hline 67 & vemurafenib & oxiconazole & erlotinib & tolnaftate & 0.729 & 0.778 & QTC prolongation \\
\hline 68 & vemurafenib & agomelatine & erlotinib & vortioxetine & 0.729 & 0.74 & QTC prolongation \\
\hline 69 & flupentixol & lopinavir & perphenazine & amprenavir & 0.779 & 0.733 & QTc prolongation \\
\hline 70 & disopyramide & ibutilide & aprindine & flecainide & 0.794 & 0.76 & QTc prolongation \\
\hline 71 & citalopram & sotalol & clomipramine & flecainide & 0.773 & 0.803 & QTC prolongation \\
\hline 72 & citalopram & ibutilide & reboxetine & flecainide & 0.731 & 0.76 & QTC prolongation \\
\hline 73 & disopyramide & zuclopenthixol & aprindine & fluphenazine & 0.794 & 0.73 & QTC prolongation \\
\hline 74 & rasagiline & methyldopa & entacapone & hydralazine & 0.751 & 0.708 & Hypertension \\
\hline 75 & selegiline & methyldopa & entacapone & hydralazine & 0.761 & 0.708 & Hypertension \\
\hline 76 & isocarboxazid & methyldopa & maprotiline & hydralazine & 0.707 & 0.708 & Hypertension \\
\hline 77 & minaprine & methyldopa & maprotiline & hydralazine & 0.717 & 0.708 & Hypertension \\
\hline 78 & phenelzine & methyldopa & maprotiline & hydralazine & 0.714 & 0.708 & Hypertension \\
\hline 79 & ranylcypromine & methyldopa & maprotiline & hydralazine & 0.728 & 0.708 & Hypertension \\
\hline 80 & moclobemide & methyldopa & nefazodone & hydralazine & 0.787 & 0.708 & Hypertension \\
\hline 81 & pargyline & methyldopa & nialamide & hydralazine & 0.798 & 0.708 & Hypertension \\
\hline 82 & quinidine & asenapine & mexiletine & lurasidone & 0.732 & 0.817 & QTC prolongation \\
\hline 83 & moclobemide & atomoxetine & nefazodone & methylphenidate & 0.787 & 0.795 & Central neurotoxicity \\
\hline 84 & disopyramide & quinidine & aprindine & mexiletine & 0.794 & 0.732 & QTc prolongation \\
\hline 85 & cisapride & quinidine & domperidone & mexiletine & 0.726 & 0.732 & QTC prolongation \\
\hline 86 & flupentixol & quinidine & perphenazine & mexiletine & 0.779 & 0.732 & QTc prolongation \\
\hline 87 & disopyramide & flupentixol & aprindine & perphenazine & 0.794 & 0.779 & QTC prolongation \\
\hline 88 & cisapride & flupentixol & tegaserod & perphenazine & 0.725 & 0.779 & QTc prolongation \\
\hline 89 & cisapride & zuclopenthixol & tegaserod & perphenazine & 0.725 & 0.725 & QTc prolongation \\
\hline 90 & flupentixol & nilotinib & perphenazine & ponatinib & 0.779 & 0.771 & QTc prolongation \\
\hline 91 & disopyramide & iloperidone & aprindine & sertindole & 0.794 & 0.753 & QTc prolongation \\
\hline 92 & cisapride & iloperidone & tegaserod & sertindole & 0.725 & 0.753 & QTc prolongation \\
\hline 93 & amiloride & tacrolimus & bumetanide & sirolimus & 0.761 & 0.74 & Hyperkalemia \\
\hline 94 & triamterene & tacrolimus & chlorothiazide & sirolimus & 0.806 & 0.74 & Hyperkalemia \\
\hline 95 & disopyramide & pimozide & aprindine & sulpiride & 0.794 & 0.738 & QTC prolongation \\
\hline 96 & disopyramide & cisapride & aprindine & tegaserod & 0.794 & 0.725 & QTc prolongation \\
\hline 97 & disopyramide & asenapine & aprindine & vilazodone & 0.794 & 0.718 & QTc prolongation \\
\hline 98 & cisapride & asenapine & tegaserod & vilazodone & 0.725 & 0.718 & QTc prolongation \\
\hline 99 & teniposide & vincristine & fotemustine & vinblastine & 0.705 & 0.788 & Neurotoxicity \\
\hline
\end{tabular}

the application of the Glay Algorithm on the drug-drug network to partition it into clusters of mostly related drugs. As a result of applying the Glay clustering algorithm on the pruned DDI graph, 110 clusters with an average member size 8 and an average clustering coefficient of 0.445 are generated. We made the result of this clustering available on GitHub [49].

ADDI uses the result of clustering to recommend alternatives (for details see Section 2.3). As a result, ADDI discovered 99 out of 180 DDIs with NHETs that fulfill all constraints regarding recommending alternatives. The 99 DDIs with NHETs, together with their alternatives, are shown in Table 4 and are also available at [49]. We used SIDER to exclude drugs which have the same side effect as an NHET. In Table 4, different colors represent the common drug classes, each of which is shown in Table 5.

\section{Discussion}

A more detailed look into Table 4 shows that most of the NHETs are QTc prolongations. QTc prolongation is a known side effect of many drug groups, including Class Ia and Class III antiarrhythmics, antipsychotics, and kinase inhibitors $[64,65]$. It can lead to life-threatening torsade de points tachycardia. Class Ia antiarrhythmics are sodium channel blockers that lengthen the heart's action potential leading to QT interval elongation [66]. Class III antiarrhythmics lengthen the hearth's action potential as well as making the repolarisation longer through inhibiting the potassium channels. These antiarrhythmics treat ventricular tachycardias. ADDI suggested using various type Ib and Ic antiarrhythmics, such as using flecainide (Ib) or mexiletine (Ic). Class Ib drugs shorten the action potential of the hearth; meanwhile, Ic type
Table 5

Common drug classes with colors selected to display them in Table 4.

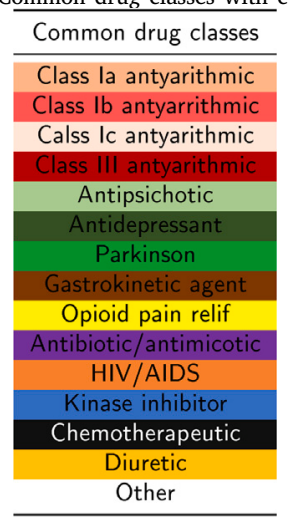

of drugs do not change it; only make the depolarization slower [66]. Flecainide is indicated for use in atrial fibrillation and supraventricular tachycardias, but clinical trials suggested it's use in catecholaminergic polymorphic ventricular tachycardia, a genetic heart disease [67]. Mexiletine is used as well for the treatment of ventricular tachycardias. In the case of antipsychotics [68] and antidepressants [69], many such drugs can have QT interval elongation effects. ADDI suggested avoiding such drugs e.g., lurasidone instead asenapine (line 82) or clomipramine instead of citalopram (line 71). In the case of any antiarrhythmic treatments with depression or psychosis, ADDI can be a useful tool to find alternative drugs for patients. In the case of the B-RAF 
inhibitor vemurafenib, ADDI suggested the EGFR inhibitor erlotinib. Vemurafenib is used in B-RAF mutant melanoma treatment [70]. Targeting EGFR besides B-RAF can increase the sensitivity of the tumors to vemurafenib [71], but erlotinib cannot replace vemurafenib.

Among the NHETs, besides QT elongation, are hypertension, hyperkalemia, and neurotoxicity. In the case of hypertension, ADDI suggested the use of hydrazidine instead of methyldopa. Both methyldopa and hydralazine are used primarily in hypertonic crisis and preeclampsia. The potential hypertonia causing interaction between methyldopa and monoamine oxidase inhibitor antidepressants starts from the inhibition of the methyldopa metabolizing monoamine oxidase. Methyldopa can form dopamine and noradrenaline, which causes hypertension [72]. This is the exact opposite of the desired therapeutic outcome. ADDI was able to find this DDI and suggest better alternatives e.g., the tetracyclic antidepressant maprotiline instead of the monoaminoxidase inhibitor minaprine.

In this case, the semantic similarity was inadequate for a replacement drug. In the case of hyperkalemia, ADDI suggested the use of loop (bumetanide) or thiazide (chlorothiazide) diuretics instead of the potassium-sparing diuretics amiloride and triamterene [73].

In the case of Neurotoxicity, ADDI suggested the use of the topoisomerase blocker teniposide [74] and the vinca alkaloid vincristine the use of fotemustine, a guanine alkylating agent [75] and vinblastine another vinca alkaloid [76] instead. Vincristine can exist at higher concentration into the central neuron system compared to vinblastine in rats [77]. Such chemotherapeutic regimes and a combination of them need careful evaluation. ADDI can flag the chemotherapeutic agents with major NHETs to consider alternatives, e.g., in the case of vincristine and vinblastine.

In conclusion, ADDI was able to recommend various therapeutic considerations, e.g., in the case of diuretics or antipsychotics, however the therapeutic indications overwrite the recommendations like in the case of vemurafenib. ADDI was able to find such NHETs where none of the drugs individually have the side effect as in the case of monoamineoxidase inhibitors and methyldopa. After finding this NHET, ADDI recommended alternative drugs to avoid discovered NHET. In the case of antiarrhythmics, the drugs' side effect and the desired effect match, so to avoid a potentially fatal side effect, proper dosage is required. Nonetheless, ADDI recommendations of the use of non-QT intervals or increasing antidepressants, or antipsychotics in combination with antiarrhythmics can be a useful tool for flagging life-threatening NHETs.

\section{Conclusions and future work}

Recently, the use of Drug-Drug Interactions (DDIs) to recommend alternative drugs has attracted much attention. To the best of our knowledge, there is no method available to use online text resources for this purpose. In this paper we proposed ADDI as such a method. ADDI applies Word2vec on text resources to extract drug embeddings and uses deep learning to predict DDI types. For the empirical results presented here, 29 million PubMed paper abstracts [45] were used as ADDI's text input. The experimental results show that, in terms of accuracy, ADDI outperforms the state-of-the-art methods by $13 \%$ on average, and it does so by using online text resources only. ADDI generates a drug-drug network and uses a clustering algorithm to recommend plausible alternatives for DDIs with negative health effects.

Regarding future research induced by our work, we see three particularly important and very promising directions for refinement and extension of our approach. First, to consider additional data available for drugs (such as chemical data and side effects) as input of ADDI that can be represented by a vector for predicting DDIs. Second, to extend ADDI towards detecting drug combinations (i.e., synergistic or antagonistic interactions) in the drug-drug interactions network. Third, to use the DDI network proposed by ADDI to discover novel relationships among drug side-effects. The main hypothesis to be evaluated is to check if two connected drugs in DDI network share same set of side-effects. We are currently focussing on the use of additional data.

\section{Declaration of competing interest}

The authors declare that they have no known competing financial interests or personal relationships that could have appeared to influence the work reported in this paper.

\section{Acknowledgment}

Thanks to Agatha Treveil for proofreading the article.

\section{References}

[1] M. Vazquez, M. Krallinger, F. Leitner, A. Valencia, Text mining for drugs and chemical compounds: methods, tools and applications, Mol. Inform. 30 (6-7) (2011) 506-519.

[2] H. Sampathkumar, B. Luo, X.-w. Chen, Mining adverse drug side-effects from online medical forums, in: 2012 IEEE Second International Conference on Healthcare Informatics, Imaging and Systems Biology, IEEE, 2012, p. 150.

[3] H. Wang, Q. Gu, J. Wei, Z. Cao, Q. Liu, Mining drug-disease relationships as a complement to medical genetics-based drug repositioning: Where a recommendation system meets genome-wide association studies, Clin. Pharmacol. Ther. 97 (5) (2015) 451-454.

[4] B. Percha, Y. Garten, R.B. Altman, Discovery and explanation of drug-drug interactions via text mining, in: Biocomputing 2012, World Scientific, 2012, pp. 410-421.

[5] L. Tari, S. Anwar, S. Liang, J. Cai, C. Baral, Discovering drug-drug interactions: a text-mining and reasoning approach based on properties of drug metabolism, Bioinformatics 26 (18) (2010) i547-i553.

[6] C. Andronis, A. Sharma, V. Virvilis, S. Deftereos, A. Persidis, Literature mining, ontologies and information visualization for drug repurposing, Brief. Bioinform. 12 (4) (2011) 357-368.

[7] R. Harpaz, A. Callahan, S. Tamang, Y. Low, D. Odgers, S. Finlayson, K. Jung, P. LePendu, N.H. Shah, Text mining for adverse drug events: the promise, challenges, and state of the art, Drug Saf. 37 (10) (2014) 777-790.

[8] M. Krallinger, R.A.-A. Erhardt, A. Valencia, Text-mining approaches in molecular biology and biomedicine, Drug Discovery Today 10 (6) (2005) 439-445.

[9] R. Feldman, J. Sanger, The Text Mining Handbook: Advanced Approaches in Analyzing Unstructured Data, Cambridge university press, 2007.

[10] V. Gupta, G.S. Lehal, et al., A survey of text mining techniques and applications, J. Emerg. Technol. Web Intell. 1 (1) (2009) 60-76.

[11] Y.-H. Tseng, C.-J. Lin, Y.-I. Lin, Text mining techniques for patent analysis, Inf. Process. Manage. 43 (5) (2007) 1216-1247

[12] C.C. Aggarwal, C. Zhai, Mining Text Data, Springer Science \& Business Media, 2012.

[13] A. Hotho, A. Nürnberger, G. Paaß, A brief survey of text mining, in: Ldv Forum, Vol. 20, Citeseer, 2005, pp. 19-62.

[14] G. Miner, J. Elder IV, A. Fast, T. Hill, R. Nisbet, D. Delen, Practical Text Mining and Statistical Analysis for Non-Structured Text Data Applications, Academic Press, 2012

[15] A. Lamurias, F.M. Couto, Text mining for bioinformatics using biomedical literature, in: Encyclopedia of Bioinformatics and Computational Biology, Vol. 1, Elsevier, 2019.

[16] J.-D. Kim, T. Ohta, Y. Tateisi, J. Tsujii, GENIA corpus-a semantically annotated corpus for bio-textmining, Bioinformatics 19 (suppl_1) (2003) i180-i182.

[17] D. Kwon, S. Kim, C.-H. Wei, R. Leaman, Z. Lu, EzTag: tagging biomedical concepts via interactive learning, Nucleic Acids Res. 46 (W1) (2018) W523-W529.

[18] D.S. Aschenbrenner, S.J. Venable, Drug Therapy in Nursing, Lippincott Williams \& Wilkins, 2009.

[19] https://aidsinfo.nih.gov/understanding-hiv-aids/fact-sheets/21/95/what-is-adrug-interaction-.

[20] https://www.pharmacologyeducation.org/clinical-pharmacology/drug interactions.

[21] https://www.rxlist.com/drug-interaction-checker.htm.

[22] L. Chen, C. Chu, Y.-H. Zhang, M. Zheng, L. Zhu, X. Kong, T. Huang, Identification of drug-drug interactions using chemical interactions, Curr. Bioinform. 12 (6) (2017) 526-534

[23] https://www.sciencedirect.com/topics/pharmacology-toxicology-andpharmaceutical-science/drug-interaction.

[24] J.Y. Ryu, H.U. Kim, S.Y. Lee, Deep learning improves prediction of drug-drug and drug-food interactions, Proc. Natl. Acad. Sci. 115 (18) (2018) E4304-E4311.

[25] B. Boyd, S. Smith, A. Gammaitoni, B.S. Galer, G.M. Farfel, A phase I, randomized, open-label, single-dose, 3-period crossover study to evaluate the drug-drug interaction between ZX008 (fenfluramine $\mathrm{HCl}$ oral solution) and a regimen of stiripentol, clobazam, and valproate in healthy subjects, Int. J. Clin. Pharmacol. Ther. 57 (1) (2019) 11.

[26] S.K. Quinney, Opportunities and challenges of using big data to detect drug-drug interaction risk, Clin. Pharmacol. Ther. 106 (1) (2019) 72. 
[27] S. Vilar, E. Uriarte, L. Santana, N.P. Tatonetti, C. Friedman, Detection of drugdrug interactions by modeling interaction profile fingerprints, PLoS One 8 (3) (2013) e58321

[28] F. Cheng, Z. Zhao, Machine learning-based prediction of drug-drug interactions by integrating drug phenotypic, therapeutic, chemical, and genomic properties, J. Am. Med. Inform. Assoc. 21 (e2) (2014) e278-e286.

[29] S. Vilar, R. Harpaz, E. Uriarte, L. Santana, R. Rabadan, C. Friedman, Drug-drug interaction through molecular structure similarity analysis, J. Am. Med. Inform. Assoc. 19 (6) (2012) 1066-1074.

[30] A. Gottlieb, G.Y. Stein, Y. Oron, E. Ruppin, R. Sharan, INDI: a computational framework for inferring drug interactions and their associated recommendations, Mol. Syst. Biol. 8 (1) (2012).

[31] P. Zhang, F. Wang, J. Hu, R. Sorrentino, Label propagation prediction of drug-drug interactions based on clinical side effects, Sci. Rep. 5 (2015) 12339.

[32] N. Rohani, C. Eslahchi, Drug-drug interaction predicting by neural network using integrated similarity, Sci. Rep. 9 (1) (2019) 1-11.

[33] N. Rohani, C. Eslahchi, A. Katanforoush, ISCMF: Integrated similarity-constrained matrix factorization for drug-drug interaction prediction, Netw. Model. Anal. Health Inform. Bioinform. 9 (1) (2020) 1-8.

[34] C. Yan, G. Duan, Y. Zhang, F. Wu, Y. Pan, J. Wang, Predicting drug-drug interactions based on integrated similarity and semi-supervised learning, IEEE/ACM Trans. Comput. Biol. Bioinform. (2020).

[35] F. Cheng, I.A. Kovács, A.-L. Barabási, Network-based prediction of drug combinations, Nat. Commun. 10 (1) (2019) 1197.

[36] S. Qian, S. Liang, H. Yu, Leveraging genetic interactions for adverse drug-drug interaction prediction, PLoS Comput. Biol. 15 (5) (2019) e1007068.

[37] M.R. Karim, M. Cochez, J.B. Jares, M. Uddin, O. Beyan, S. Decker, Drug-drug interaction prediction based on knowledge graph embeddings and convolutionalLSTM network, in: Proceedings of the 10th ACM International Conference on Bioinformatics, Computational Biology and Health Informatics, ACM, 2019, pp. $113-123$

[38] D. Huang, Z. Jiang, L. Zou, L. Li, Drug-drug interaction extraction from biomedical literature using support vector machine and long short term memory networks, Inform. Sci. 415 (2017) 100-109.

[39] Z. Zhao, Z. Yang, L. Luo, H. Lin, J. Wang, Drug drug interaction extraction from biomedical literature using syntax convolutional neural network, Bioinformatics 32 (22) (2016) 3444-3453.

[40] S. Lim, K. Lee, J. Kang, Drug drug interaction extraction from the literature using a recursive neural network, PLoS One 13 (1) (2018)

[41] J.-Y. Shi, K.-T. Mao, H. Yu, S.-M. Yiu, Detecting drug communities and predicting comprehensive drug-drug interactions via balance regularized semi-nonnegative matrix factorization, J. Cheminform. 11 (1) (2019) 1-16.

[42] Y. Zheng, H. Peng, X. Zhang, Z. Zhao, X. Gao, J. Li, DDI-PULearn: a positiveunlabeled learning method for large-scale prediction of drug-drug interactions, BMC Bioinform. 20 (19) (2019) 1-12.

[43] T. Mikolov, K. Chen, G. Corrado, J. Dean, Efficient estimation of word representations in vector space, 2013, arXiv preprint arXiv:1301.3781.

[44] L. Ma, Y. Zhang, Using word2vec to process big text data, in: 2015 IEEE International Conference on Big Data (Big Data), IEEE, 2015, pp. 2895-2897.

[45] https://ftp.ncbi.nlm.nih.gov/pubmed/baseline/, 2018.

[46] M. Allahgholi, www.github.com/miladallahgholi95/DDREL, 2019.

[47] A.B. Nassif, D. Ho, L.F. Capretz, Towards an early software estimation using log-linear regression and a multilayer perceptron model, J. Syst. Softw. 86 (1) (2013) 144-160.

[48] D.S. Wishart, Y.D. Feunang, A.C. Guo, E.J. Lo, A. Marcu, J.R. Grant, T. Sajed, D. Johnson, C. Li, Z. Sayeeda, et al., DrugBank 5.0: a major update to the DrugBank database for 2018, Nucleic Acids Res. 46 (D1) (2018) D1074-D1082.

[49] M. Allahgholi, www.github.com/miladallahgholi95/ADDI, 2020.

[50] A. Baćac, Classification of Large-Scale Biological Annotations Using Word Embeddings Derived from Corpora of Biomedical Research Literature (Ph.D. thesis), Fakultet Elektrotehnike i Računarstva, Sveučilište u Zagrebu, 2017.

[51] B. Chiu, G. Crichton, A. Korhonen, S. Pyysalo, How to train good word embeddings for biomedical NLP, in: Proceedings of the 15th Workshop on Biomedical Natural Language Processing, 2016, pp. 166-174.

[52] O. Levy, Y. Goldberg, I. Dagan, Improving distributional similarity with lessons learned from word embeddings, Trans. Assoc. Comput. Linguist. 3 (2015) 211-225.

[53] H. Caselles-Dupré, F. Lesaint, J. Royo-Letelier, Word2vec applied to recommendation: Hyperparameters matter, in: Proceedings of the 12th ACM Conference on Recommender Systems, ACM, 2018, pp. 352-356.
[54] J. Pennington, R. Socher, C. Manning, Glove: Global vectors for word representation, in: Proceedings of the 2014 Conference on Empirical Methods in Natural Language Processing (EMNLP), 2014, pp. 1532-1543.

[55] S. Lai, K. Liu, S. He, J. Zhao, How to generate a good word embedding, IEEE Intell. Syst. 31 (6) (2016) 5-14.

[56] M. TH, S. Sahu, A. Anand, Evaluating distributed word representations for capturing semantics of biomedical concepts, in: Proceedings of BioNLP 15, 2015, pp. 158-163.

[57] T. Schnabel, I. Labutov, D. Mimno, T. Joachims, Evaluation methods for unsupervised word embeddings, in: Proceedings of the 2015 Conference on Empirical Methods in Natural Language Processing, 2015, pp. 298-307.

[58] L. Chen, F. Yuan, J.M. Jose, W. Zhang, Improving negative sampling for word representation using self-embedded features, in: Proceedings of the Eleventh ACM International Conference on Web Search and Data Mining, ACM, 2018, pp. 99-107.

[59] Y. Goldberg, Neural network methods for natural language processing, Synth. Lect. Human Lang. Technol. 10 (1) (2017) 1-309.

[60] A. Santoro, D. Raposo, D.G. Barrett, M. Malinowski, R. Pascanu, P. Battaglia, T. Lillicrap, A simple neural network module for relational reasoning, in: Advances in Neural Information Processing Systems, 2017, pp. 4967-4976.

[61] J. Han, C. Moraga, The influence of the sigmoid function parameters on the speed of backpropagation learning, in: International Workshop on Artificial Neural Networks, Springer, 1995, pp. 195-201.

[62] I. Goodfellow, Y. Bengio, A. Courville, Deep Learning, MIT press, 2016.

[63] G. Su, A. Kuchinsky, J.H. Morris, D.J. States, F. Meng, Glay: community structure analysis of biological networks, Bioinformatics 26 (24) (2010) 3135-3137.

[64] S. Nachimuthu, M.D. Assar, J.M. Schussler, Drug-induced QT interval prolongation: mechanisms and clinical management, Ther. Adv. Drug Saf. 3 (5) (2012) 241-253.

[65] C.V. Etchegoyen, G.A. Keller, S. Mrad, S. Cheng, G. Di Girolamo, Drug-induced QT interval prolongation in the intensive care unit, Curr. Clin. Pharmacol. 12 (4) (2017) 210-222

[66] G.M. Chaudhry, C.I. Haffajee, Antiarrhythmic agents and proarrhythmia, Crit. Care Med. 28 (10) (2000) N158-N164.

[67] P.J. Kannankeril, J.P. Moore, M. Cerrone, S.G. Priori, N.J. Kertesz, P.S. Ro, A.S Batra, E.S. Kaufman, D.L. Fairbrother, E.V. Saarel, et al., Efficacy of flecainide in the treatment of catecholaminergic polymorphic ventricular tachycardia: a randomized clinical trial, JAMA Cardiol. 2 (7) (2017) 759-766.

[68] M. Huhn, A. Nikolakopoulou, J. Schneider-Thoma, M. Krause, M. Samara, N. Peter, T. Arndt, L. Bäckers, P. Rothe, A. Cipriani, et al., Comparative efficacy and tolerability of 32 oral antipsychotics for the acute treatment of adults with multiepisode schizophrenia: a systematic review and network meta-analysis, Lancet 394 (10202) (2019) 939-951.

[69] M.P. Rochester, A.M. Kane, S.A. Linnebur, D.R. Fixen, Evaluating the risk of QTc prolongation associated with antidepressant use in older adults: a review of the evidence, Ther. Adv. Drug Saf. 9 (6) (2018) 297-308.

[70] P.B. Chapman, A. Hauschild, C. Robert, J.B. Haanen, P. Ascierto, J. Larkin, R. Dummer, C. Garbe, A. Testori, M. Maio, et al., Improved survival with vemurafenib in melanoma with BRAF V600E mutation, New Engl. J. Med. 364 (26) (2011) 2507-2516.

[71] I. Kenessey, Z. Kramer, L. István, M.T. Cserepes, T. Garay, B. Hegedús, J. Dobos, J. Tímár, J. Tóvári, Inhibition of epidermal growth factor receptor improves antitumor efficacy of vemurafenib in BRAF-mutant human melanoma in preclinical model, Melanoma Res. 28 (6) (2018) 536-546.

[72] V. Rossumj, J. Hurkmans, Reversal of the effect of alpha-methyldopa by monoamine oxidase inhibitors, J. Pharm. Pharmacol. 15 (1963) 493-499.

[73] D.L. Blowey, Diuretics in the treatment of hypertension, Pediatr. Nephrol. 31 (12) (2016) 2223-2233.

[74] P.I. Clark, M.L. Slevin, The clinical pharmacology of etoposide and teniposide, Clin. Pharmacokinet. 12 (4) (1987) 223-252.

[75] A. De Rossi, L. Rossi, A. Laudisi, V. Sini, L. Toppo, F. Marchesi, G. Tortorelli, M. Leti, M. Turriziani, A. Aquino, et al., Focus on fotemustine, J. Exp. Clin. Cancer Res. 25 (4) (2006) 461.

[76] S. Florian, T.J. Mitchison, Anti-microtubule drugs, in: The Mitotic Spindle, Springer, 2016, pp. 403-421.

[77] N.H. Greig, T.T. Soncrant, H.U. Shetty, S. Momma, Q.R. Smith, S.I. Rapoport, Brain uptake and anticancer activities of vincristine and vinblastine are restricted by their low cerebrovascular permeability and binding to plasma constituents in rat, Cancer Chemother. Pharmacol. 26 (4) (1990) 263. 